\title{
CONTRIBUTIONS OF THE ELASTICITY TO THE PRECESSION OF A TWO-LAYER EARTH MODEL
}

\author{
T. Baenas ${ }^{1}$, J. M. Ferrándiz ${ }^{1}$, A. Escapa ${ }^{1,2}$, J. Getino ${ }^{3}$, and J. F. Navarro ${ }^{1}$ \\ ${ }^{1}$ Department of Applied Mathematics, University of Alicante, PO Box 99, E-03080 Alicante, Spain; Tomas.Baenas@ua.es \\ ${ }^{2}$ Department of Aerospace Engineering, University of León, E-24071 León, Spain \\ ${ }^{3}$ Department of Applied Mathematics, University of Valladolid, E-47011 Valladolid, Spain \\ Received 2016 July 28; revised 2016 December 21; accepted 2016 December 22; published 2017 January 25
}

\begin{abstract}
We focus on the updating of a specific contribution to the precession of the equator in longitude, usually named as "second order." It stems from the crossing of certain terms of the lunisolar gravitational potential. The IAU2006 precession theory assigns it the value of $-46.8 \mathrm{mas} / \mathrm{cy}$ that was derived for a rigid Earth model. Instead of that model, we consider a two-layer Earth composed of an elastic mantle and a liquid core, working out the problem within the Hamiltonian framework developed by Getino and Ferrándiz. The targeted effect is obtained without further simplifying assumptions through Hori's canonical perturbation method applied up to the second order of perturbation. On account of using a more realistic Earth model, the revised value of the second-order contribution is significantly changed and reaches $-55.29 \mathrm{mas} / \mathrm{cy}$. That variation of the second-order contribution is larger than other contributions included in IAU2006. It must be compensated with an increase of -8.51 mas/cy in the value of the lunisolar first-order component $p_{A}^{\prime}$ of the precession of the equator rate, which is derived from the total rate by subtracting the remaining contributions accounted for in IAU2006 precession. The updating of the second-order contribution implies that the $p_{A}^{\prime}$ parameter has to be changed, from 5040684.593 to $5040693.104 \mathrm{mas} / \mathrm{cy}$ in absence of potential revisions of other contributions. It entails a proportional variation of Earth's dynamical ellipticity $H_{d}$, for which the estimation associated with IAU2006, 0.00327379448, should be updated to 0.00327380001 , about 1.7 ppm larger.
\end{abstract}

Key words: celestial mechanics - Earth - methods: analytical - reference systems

\section{INTRODUCTION}

The main constituent of the precession of Earth's equator longitude is a linear variation due to the first-order effect of the lunisolar torque. Besides, there are additional contributions to the longitude rate coming from other sources. Most of the contributions considered in the International Astronomical Union (IAU) precession theories IAU1976 and IAU2006 are computed for a rigid Earth model. However, Earth's internal structure also originates contributions of non-negligible magnitude that cannot be ignored henceforward given the current accuracy of the observations and the stringent accuracy and stability demanded for the reference frames.

The theoretical modeling of the precession has advanced significantly over the past decades following the increasingly accurate monitoring of Earth's rotation by the very long baseline interferometry (VLBI) technique. In the comprehensive revision of the precession theory carried out by Fukushima (2003) for developing a new set of precession formulae, the speed of the general precession in longitude $\left(p_{A}\right)$ was estimated as $p=5028.7958 \pm 0$ ". $0003 /$ cy (where cy stands for Julian century). That uncertainty of $300 \mu \mathrm{as} / \mathrm{cy}$, equivalent to a relative error of about $60 \mathrm{ppb}$ (parts per billion), is of the same order of magnitude as the targets of accuracy and stability of the reference frames established by the Global Geodetic Observing System (GGOS) of the International Association of Geodesy (IAG), namely, $1 \mathrm{~mm}$ in position and $1 \mathrm{~mm} \mathrm{yr}^{-1}$ in stability (Plag \& Pearlman 2009). Those goals, which correspond to geocentric angles and angular velocities near 30 $\mu$ as and $300 \mu \mathrm{as} / \mathrm{cy}$, were adopted by the IAU/IAG Joint Working Group on Theory of Earth Rotation in 2013 (see, e.g., Ferrándiz \& Gross 2015).

In 2006 (Hilton et al. 2006) the General Assembly of the IAU approved Resolution 1, which endorsed the P03 model by
Capitaine et al. $(2003,2005)$ as the new precession theory IAU2006 that superseded Lieske's (1977) IAU1976. IAU2006 uses fifth-degree polynomials to model the precession variables of the equator and the ecliptic. This kind of polynomial expansion is the most used in precession theories (see, however, Vondrák et al. 2011). The rate of the longitude of the node of the equator adopted in IAU2006 is 5038". 481507/ cy. It consists of several components. By far, the largest of them, denoted here ${ }^{4}$ as $p_{A}^{\prime}$, is due to the first-order effect of the lunisolar torque acting on the oblate Earth, close to $5040^{\prime \prime} / \mathrm{cy}-$ namely, the linear approximation of the solution for MacCullagh's term of the lunisolar potential. It is given by a simple formula depending on Earth's angular velocity $\omega_{E}$ and dynamical ellipticity

$$
H_{d}=\frac{2 C-A-B}{2 C}
$$

the masses of the Sun, Moon, and Earth, and some orbital quantities (Kinoshita \& Souchay 1990, Equation (8.7))

$$
\begin{aligned}
p_{A}^{\prime}= & 3 H_{d}\left[\left(\frac{m_{M}}{m_{M}+m_{E}}\right)\left(\frac{n_{M}^{2}}{\omega_{E}}\right) M_{0}\right. \\
& \left.+\left(\frac{m_{S}}{m_{S}+m_{E}+m_{M}}\right)\left(\frac{n_{S}^{2}}{\omega_{E}}\right) S_{0}\right] \cos \epsilon_{A} .
\end{aligned}
$$

Each theory arrives at different determinations of $H_{d}$ (usually without specifying estimated errors) depending on the precession rate inferred from observations and on the remaining

\footnotetext{
4 We follow the notation introduced by Souchay \& Kinoshita (1996). Notice that this parameter is the same as that denoted $\left(r_{0}\right)_{1}$ by Capitaine et al. (2003), while, e.g., Fukushima (2003) uses $P^{\prime}$.
} 
Table 1

Different Determinations of the Dynamical Ellipticity

\begin{tabular}{lcl}
\hline \hline$H_{d}$ Value & Uncertainty & Source \\
\hline 0.0032737634 & $\ldots$ & Williams (1994) \\
0.0032737548 & $\ldots$ & REN2000, Souchay et al. (1999) \\
0.0032737674 & $\ldots$ & RDAN97, Roosbeek \& Dehant (1998) \\
0.0032737949 & $12 \times 10^{-10}$ & MHB2000, Mathews et al. (2002) \\
0.0032737804 & $3 \times 10^{-10}$ & Fukushima (2003) \\
0.00327379448 & $\ldots$ & P03, Capitaine et al. (2003) \\
0.003273796 & $\ldots$ & FNEG04, Ferrándiz et al. (2004) \\
0.00327380001 & $\ldots$ & This work \\
\hline
\end{tabular}

Note. Ellipsis points means that the uncertainty is not provided in the source along with the value. The $H_{d}$ value in MHB2000 is $12 \mathrm{ppm}$ larger than the corresponding parameter in its underlying rigid Earth theory REN2000.

theoretical contributions accounted for. A selection of values and their sources are displayed in Table 1.

Let us recall that the dynamical ellipticity is a main parameter that also factorizes all the first-order nutation terms. Therefore, any change of the adopted $H_{d}$ requires correcting the nutation amplitudes by computing the so-called indirect effects (Escapa et al. 2016).

Besides the main lunisolar first-order rate of the equator longitude, the IAU2006 precession theory included many effects of noticeably smaller magnitude than $p_{A}^{\prime}$, which are listed in Table 3 of Capitaine et al. (2003) - with an update of a single component in Capitaine et al. (2005). Most of them were derived before the adoption of the MHB2000 theory (Mathews et al. 2002) as the IAU2000 nutation theory, and their values are practically the same as given by, e.g., Kinoshita \& Souchay (1990), Williams (1994, 1995), or Souchay \& Kinoshita (1996), despite the simplifying assumptions made in some of the former theoretical models. The contributions included in IAU2006 (Capitaine et al. 2003, 2005) are listed in Table 2. All of them but the last one are derived for a rigid Earth model. The largest contribution is 400 parts per million $(\mathrm{ppm})$ of $p_{A}^{\prime}$, due to the geodesic precession.

However, new contributions to the precession rate have been found since the P03 derivation (e.g., Liu \& Capitaine 2015). For this paper's sake, we emphasize the generalization of the IAU2006 (REN2000) lunisolar second-order effects to a nonrigid Earth (Ferrándiz et al. 2004). The contribution to the longitude rate that IAU2006 names as lunisolar second-order effect (split in one part for the Moon and another for the Sun, labeled "a" and "b" respectively) comes from the main (zonal) terms of the second order of perturbation solution of the rigid Earth Hamiltonian (quadratic in $H_{d}$ ); it was derived by Kinoshita \& Souchay (1990) and remained unchanged in REN2000. Ferrándiz et al. (2004) showed that, unlike commonly believed, the presence of a fluid core affects the precessional motion of Earth by a non-negligible amount, reaching $-5 \mathrm{ppm}$ of the value of the precession constant when a Poincaré-like Earth model (rigid mantle with fluid core) is assumed. An explanation can be found in the amplification of the Oppolzer terms caused by the resonance associated with the core.

This research consists in a further extension of those lunisolar second-order effects for a two-layer Earth model (Ferrándiz et al. 2004) by including the contributions of the elasticity to the precession in longitude. Some partial and preliminary computations of those contributions were derived by Ferrándiz et al. (2007), but only the numerical results were presented, due to the nature of that concise proceedings report.
Table 2

Contributions to Precession Rate Included in IAU2006

\begin{tabular}{lrl}
\hline \hline Contribution & Value (mas/cy) & Source \\
\hline First-order lunisolar $\left(p_{A}^{\prime}\right)^{\mathrm{a}}$ & 5040684.593 & Capitaine et al. (2005) \\
Second-order lunisolar & -46.780 & Williams (1994) \\
Lunisolar $J_{4}$ & +2.600 & Williams (1994) \\
First-order planetary & +31.367 & Capitaine et al. (2003) \\
$J_{2}$-tilt & -269.430 & Williams (1994) \\
Geodesic precession & -1919.883 & Capitaine et al. (2003) \\
MHB2000's nonlinear effect & -0.960 & Mathews (unpublished) \\
\hline
\end{tabular}

Notes.

${ }^{\mathrm{a}}$ Not provided explicitly, but obtained by subtraction.

${ }^{\mathrm{b}}$ Recomputation of the Mathews et al. (2002) value cited in Capitaine et al. (2005).

Note that these terms are of a different nature from those related with the redistribution tidal potential, and currently they cannot be provided by any theory but the Hamiltonian one presented in this work. ${ }^{5}$ Specifically, we evaluate the secondorder contributions to the precession in longitude through Hori's canonical method for a two-layer Earth model composed of an elastic mantle and a fluid core. They arise as a consequence of the crossing of first-order nutation terms due to the $(2,0)$ harmonic of the gravitational potential and the tidal rotational kinetic energy. We perform a rigorous computation and a detailed analysis of those contributions. They involve the indirect terms and also the direct ones, which must be incorporated when fully taking into account the influence of the elasticity of the model and nowadays accuracy requirements. The indirect terms reflect the effect of the core through the nearly diurnal free wobble (NDFW), i.e., they can be associated with the free core nutation (FCN), whereas the direct ones stem from an additional contribution to the kinetic energy of the system entirely due to the fluid core.

The structure of the paper is as follows. Section 2 contains an outline of Hori's canonical method that is applied to the Hamiltonian of the non-rigid Earth model considered in this work. It provides analytical expressions for the second-order contributions to the precession rate. Those expressions are split according to their different origins, which is possible thanks to the analytical approach used in their derivation. Section 3 contains a numerical evaluation of new contributions, comparing them with other relevant second-order corrections appearing in the literature. Finally, in Section 4, we draw the main conclusions of this investigation. The paper is completed with two appendices including some auxiliary material.

\section{DERIVATION OF THE NEW SECOND-ORDER CONTRIBUTION TO PRECESSION}

The solution that contains the new contribution to the precession, due to the previously mentioned non-rigid Earth structure, is worked out using the Hamiltonian approach developed by Getino \& Ferrándiz (1991, 1997, 2001) for the non-rigid Earth. Some of its main features are as follows: (1) it allows a consistent treatment, since the first- and second-order lunisolar perturbations are derived from a sole Hamiltonian, with only a set of basic Earth parameters (BEPs) and a sole

\footnotetext{
5 The nonlinear effects presented in Mathews et al. (2002) are related to some part of the redistribution tidal potential (Lambert \& Mathews 2006), i.e., to the effects of the variation of the geopotential due to tidal deformations.
} 
tide-generating expansion; (2) the crossing effects are rigorously obtained by Hori's (1966) perturbation method; and (3) the procedure is systematic and can be performed by computer algebra; therefore, no group of terms must be neglected in advance to simplify the analytical derivations (unlike what happens usually in less elaborated approaches dealing with the equations of motion).

\subsection{Outline of the Method}

Next, the procedure followed in the derivation is sketched. The method is very close to that used in Ferrándiz et al. (2004) to derive an analogous contribution to the precession in the case of rigid mantle, although the consideration of the elasticity introduces noticeable complexities and differences. The original Hamiltonian, $\mathcal{H}$, is divided into perturbation orders and then transformed into a new Hamiltonian, $\mathcal{K}$, easier to solve, by calculating the generating function, $\mathcal{W}$, of a certain canonical transformation close to the identity. Finally, the precession rate perturbation is computed by taking a certain partial derivative of the generating function.

More precisely, we apply a perturbation method based on Lie series up to the second order, which is equivalent to the Hori (1966) canonical method, combined with an averaging requirement. The starting ("old") and transformed ("new") Hamiltonians are each divided into an unperturbed or zerothorder term and perturbation terms of first and second orders, namely,

$$
\begin{aligned}
& \mathcal{H}=\mathcal{H}_{0}+\mathcal{H}_{1}+\mathcal{H}_{2}, \\
& \mathcal{K}=\mathcal{K}_{0}+\mathcal{K}_{1}+\mathcal{K}_{2},
\end{aligned}
$$

with

$$
\mathcal{K}_{0}=\mathcal{H}_{0}, \mathcal{K}_{1}=\mathcal{H}_{1, \mathrm{sec}}, \mathcal{K}_{2}=\frac{1}{2}\left\{\mathcal{H}_{1}+\mathcal{K}_{1}, \mathcal{W}_{1}\right\}_{\mathrm{sec}}+\mathcal{H}_{2, \mathrm{sec}}
$$

Here $\{--,--\}$ denotes the Poisson bracket and subscript "sec" the secular part (or time average) of a function. The components of the generating function $\mathcal{W}=\mathcal{W}_{1}+\mathcal{W}_{2}$ satisfy the equations of the method

$$
\begin{gathered}
\left\{\mathcal{H}_{0}, \mathcal{W}_{1}\right\}=\mathcal{K}_{1}-\mathcal{H}_{1} \equiv \mathcal{A}_{1} \\
\left\{\mathcal{H}_{0}, \mathcal{W}_{2}\right\}=\mathcal{K}_{2}-\mathcal{H}_{2}-\frac{1}{2}\left\{\mathcal{H}_{1}+\mathcal{K}_{1}, \mathcal{W}_{1}\right\} \equiv \mathcal{A}_{2} .
\end{gathered}
$$

Their solutions $\mathcal{W}_{i}$ can be expressed as

$$
\mathcal{W}_{i}=-\int_{\mathrm{UP}} \mathcal{A}_{i} d t
$$

where subscript UP means that the integral is computed along the trajectories of the unperturbed problem $\left(\mathcal{H}_{0}\right)$. It is understood that functions on the right-hand side of Equations (4)-(7) are expressed in the new canonical variables $(q, p)$ on which $\mathcal{K}$ depends by literally substituting the old by the new ones (Ferraz-Mello 2007).

Notice that all the terms $\mathcal{K}_{i}$ of the new Hamiltonian are defined in order to be free from periodic components, whereas functions $\mathcal{A}_{i}$ and $\mathcal{W}_{i}$ average to zero. The method provides an asymptotic approximate solution to the original variables as the sum of periodic perturbations superposed to a secular evolution. The periodic perturbations are derived from the so-called perturbation equations and are not needed for this paper's goals. The secular motion is the solution of the transformed Hamiltonian, which is free of periodic terms up to the second order of perturbation. Therefore, the equations derived from $\mathcal{K}$ give rise to the secular motion of the canonical variables, which is the relevant one to study precession. More precisely, if the secular solution is assumed to be known up to the first order of perturbation, the additive contribution to the rate of a canonical coordinate $q_{j}$ conjugate to $p_{j}$ (e.g., the incremental precessional rate in our problem) is obtained by means of

$$
\delta\left(\frac{d q_{j}}{d t}\right)=\left\{q_{j}, \mathcal{K}_{2}\right\}=\frac{\partial \mathcal{K}_{2}}{\partial p_{j}} .
$$

\subsection{Arrangement of the Hamiltonian of the Two-layer Earth}

Let us consider a two-layer Earth composed of a mantle and a fluid core and subject to elastic deformations. A Hamiltonian formulation of that system was used by Getino \& Ferrándiz (2001) in their GF2000 nutation solution, by means of an Andoyer-like set of canonical variables. The first step is casting the Hamiltonian into the form given by Equation (3). Term $\mathcal{H}_{2}$ gathers some small perturbations, e.g., the lunisolar potential accounting for the rigid Earth's triaxiality (Kinoshita 1977) or corresponding to its higher-degree harmonics (Folgueira et al. 1998, 1999, 2001), the planetary rigid Earth potential (Kinoshita \& Souchay 1990), and non-rigid Earth terms like the additional potential due to the deformations induced by the rotational potential or lunisolar attraction (Getino \& Ferrándiz 1995). However, the $\mathcal{H}_{2}$ contribution to precession, independently of its magnitude, only depends on its secular part $\mathcal{K}_{2}$; as we are concerned here only with the effect arising from the interaction among $\mathcal{H}_{1}, \mathcal{K}_{1}$, and $\mathcal{W}_{1}$, according to Equations (4) and (8), the expression of $\mathcal{H}_{2}$ is irrelevant for our purpose and can be ignored without altering the results.

We can start from the simplified Hamiltonian

$$
\mathcal{H}=\mathcal{H}_{0}+\mathcal{H}_{1},
$$

which replaces Equation (3), where

$$
\mathcal{H}_{0}=T_{0}, \mathcal{H}_{1}=E+V_{\mathrm{sec}}+V_{\text {per }}+T_{t}
$$

In these expressions, $T_{0}$ is the rotational kinetic energy of Earth in a certain unperturbed state prior to deformation considered in the definition of the canonical variables, $E$ is the term introduced by Kinoshita (1977) for referring the problem to the ecliptic of date, and $V_{\mathrm{sec}}$ and $V_{\mathrm{per}}$ are, respectively, the secular and periodic part of the $(2,0)$ harmonic (the $J_{2}$ term) of the gravitational potential. Finally, $T_{t}$ is the increment of the rotational kinetic energy arising from the change of the inertia tensor caused by Earth's tidal deformation. This term is responsible of the contributions to the precession in longitude worked out in this investigation, and it is absent in Poincaré's Earth model (Ferrándiz et al. 2004).

With this decomposition of $\mathcal{H}$, the secular perturbation terms of Hamiltonian $\mathcal{K}$ read as

$$
\mathcal{K}_{1}=E+V_{\text {sec }}, \mathcal{K}_{2}=\frac{1}{2}\left\{\mathcal{H}_{1}+\mathcal{K}_{1}, \mathcal{W}_{1}\right\}_{\text {sec }}
$$

where

$$
\mathcal{W}_{1}=\int_{U P}\left(V_{\mathrm{per}}+T_{t}\right) d t
$$




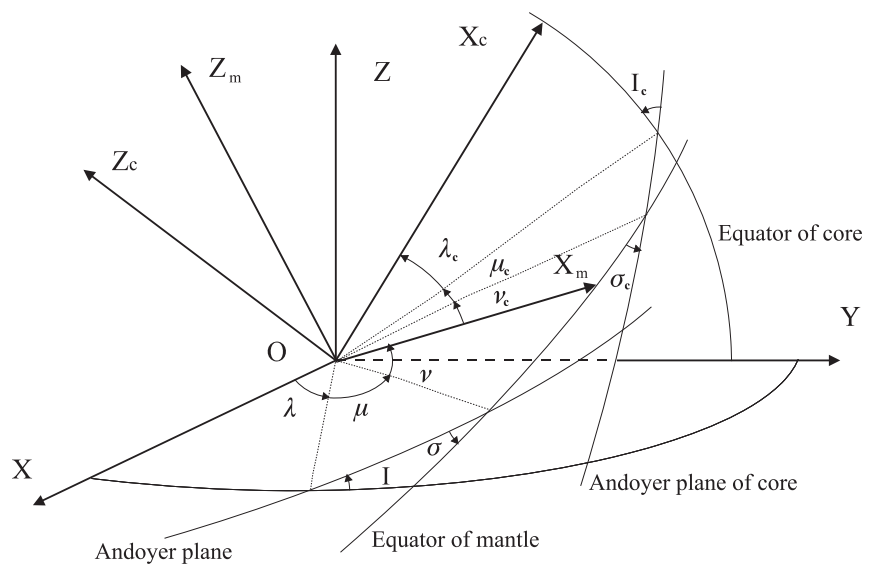

Figure 1. Andoyer-like canonical set for a two-layer Earth model.

Getino \& Ferrándiz (2001) expressed the Hamiltonian in a convenient canonical set $\left(M, N, \Lambda, M_{c}, N_{c}, \Lambda_{c} ; \mu, \nu, \lambda, \mu_{c}, \nu_{c}\right.$, $\lambda_{c}$ ), where variables with $c$ subscripts correspond to the core, and the rest to the whole Earth-see Figure 1 and also Getino (1995) or Getino \& Ferrándiz (1997) for additional details. ${ }^{6}$ Andoyer's plane (orthogonal to the total angular momentum) is defined by its longitude $\lambda$ (reckoned in the direct sense) and the auxiliary angle $I=\cos ^{-1}(\Lambda / M)$. The secular evolutions of the equatorial plane and the Andoyer plane are the same, and thus the classic precessional variables $\psi_{A}$ and $\epsilon_{A}$, which provide the longitude and obliquity of the ecliptic, are related to Andoyer's by $\psi_{A}=-\lambda$ and $\epsilon_{A}=-I$.

Therefore, the rate $p_{A}$ of the general precession in longitude is given by Getino \& Ferrándiz (1995),

$$
p_{A}=-\frac{d \lambda}{d t}=-\frac{\partial \mathcal{K}(\Lambda, I(\Lambda))}{\partial \Lambda}=-\left(\frac{\partial \mathcal{K}}{\partial \Lambda}\right)+\frac{1}{M \sin I} \frac{\partial \mathcal{K}}{\partial I} .
$$

The unperturbed term $\mathcal{K}_{0}$ does not depend on $\Lambda$ and $I$ or contribute to $p_{A}$ accordingly. The first-order contribution arising from $\mathcal{K}_{1}$ is

$$
\left.p\right|_{\mathcal{K}_{1}}=-\frac{\partial E}{\partial \Lambda}+\frac{1}{M \sin I} \frac{\partial}{\partial I}\left(E+V_{\mathrm{sec}}\right)=p_{A}^{\prime}+S_{E}^{L},
$$

where $p_{A}^{\prime}$ is the main term of the longitude rate given in Equation (2), and the contribution from the moving ecliptic $S_{E}^{L}$ is the same as derived by Getino et al. (2010, Equation (C1)) for the rigid Earth. In turn, the second-order contribution to precession in longitude is given by

$$
\left.\delta p\right|_{\mathcal{K}_{2}}=\frac{1}{M \sin I} \frac{\partial \mathcal{K}_{2}}{\partial I}
$$

with $\mathcal{K}_{2}$ being reduced to

$$
\mathcal{K}_{2}=\frac{1}{2}\left\{V_{\mathrm{per}}+T_{t}, \mathcal{W}\right\}_{\mathrm{sec}},
$$

since $\left\{\mathcal{K}_{1}, \mathcal{W}\right\}$ has no secular part. Finally, let us indicate that the only contribution to the obliquity rate under our assumptions stems from the term $E$ (Getino et al. 2010), since the

\footnotetext{
6 When reducing our model to the rigid case, the variables $(M, N, \Lambda ; \mu, \nu, \lambda)$ correspond to $(G, L, H ; g, l, h)$ in Kinoshita's notation. Similarly, the auxiliary angle, $\sigma=\cos ^{-1} N / M$, corresponds to $J$.
}

perturbation model includes no dissipations-algebraically, $\mathcal{K}_{2}$ does not depend on angle $\lambda$ since it is defined by averaging.

\subsection{Calculation of $\mathcal{K}_{2}$}

The components of the periodic first-order term of the Hamiltonian, $V_{\text {per }}+T_{t}$, appear in previous work of the authors (see, e.g., Getino \& Ferrándiz 2001, Equations (55) and (59)) and can be written as

$$
\begin{aligned}
V_{\mathrm{per}}= & \sum_{p=M, S} k_{p}^{\prime}\left[\frac{1}{2}\left(3 \cos ^{2} \sigma-1\right) \sum_{i \neq 0} B_{i, p}(I) \cos \Theta_{i}\right. \\
& \left.-\frac{1}{2} \sin 2 \sigma \sum_{i} \sum_{\tau= \pm 1} C_{i, \tau, p}(I) \cos \left(\mu-\tau \Theta_{i}\right)\right] \\
T_{t}= & \sum_{p=M, S} \sum_{i} \sum_{\tau= \pm 1} C_{i, \tau, p}(\tilde{I})\left[k_{p}^{m} M \sin \sigma \cos \left(\tilde{h}_{i, \tau}-\nu\right)\right. \\
& \left.+\left(k_{p}^{m}-k_{p}^{c}\right) M_{c} \sin \sigma_{c} \cos \left(\tilde{h}_{i, \tau}+\nu_{c}\right)\right]
\end{aligned}
$$

In the formulae above, the first summation index $p$ takes the values of the perturbing bodies, namely, $M$ (Moon) and $S$ (Sun), and will be omitted hereinafter to simplify the expressions. Constants $k_{p}^{\prime}$ are the same as used in Kinoshita's rigid Earth theory, following the notation introduced by Kinoshita \& Souchay (1990); $k_{p}^{m}$ and $k_{p}^{c}$ are the constants introduced by Getino \& Ferrándiz (2001) to account for the energy variations of mantle and core, respectively, induced by elastic deformation, which have similar units to Kinoshita's $k_{p}$ and are proportional to the $k_{2}$ Love numbers and Kubo's $\kappa$ (Kubo 1991; Escapa 2011); $B_{i, p}(I)$ and $C_{i, \tau, p}(I)$ are the orbital functions (see Appendix A) and $\Theta_{i}$ the nutation arguments used by Kinoshita (1977), which depend on a five-dimensional vector subindex $i=\left(m_{1 i}, m_{2 i}, m_{3 i}, m_{4 i}, m_{5 i}\right)$ such that

$$
\Theta_{i}=m_{1 i} l+m_{2 i} l^{\prime}+m_{3 i} F+m_{4 i} D+m_{5 i} \Omega,
$$

with $F=l+g, D=l+g+h-l^{\prime}-g^{\prime}-h^{\prime}, \Omega=h-\lambda$, and $(l, g, h),\left(l^{\prime}, g^{\prime}, h^{\prime}\right)$ the Delaunay variables of the Moon and Sun, respectively.

As for the remaining variables, the auxiliary angle $\sigma=\cos ^{-1}(N / M)$ measures the deviation between the angular momentum and figure axes, which allows neglecting the terms of $\sigma^{2}$ order that factorize semidiurnal arguments in this precession study-as done in Equation (17). Variables $h_{i, \tau}=\mu+\nu-\tau \Theta_{i}$ were introduced by Getino \& Ferrándiz (2001) and lie in the diurnal band since $d(\mu+\nu) / d t=\omega_{E}$.

Finally, when the variables specifying Earth's rotation must be considered as time-dependent functions, because Earth is playing the role of perturbing body in the elastic problem (Kaula 1964; Efroimsky \& Williams 2009), they are denoted with a tilde (e.g., $\tilde{I}, \tilde{\nu})$ alike in Getino \& Ferrándiz (1995, 2001). For the sake of simplicity, the argument of Kinoshita's functions is omitted in many steps and a self-explained abridged notation is used, e.g., $C_{i, \tau, p} \equiv C_{i, \tau, p}(I), \quad \tilde{C}_{i, \tau, p} \equiv$ $C_{i, \tau, p}(\tilde{I})$.

The first-order generating function (12) is the same as obtained in Equations (56) and (62) of Getino \& Ferrándiz (2001), particularizing them for our Earth model $(\Gamma=0)$. Therefore, we can proceed with

$$
\mathcal{W}_{1}=\mathcal{W}_{P}+\mathcal{W}_{t}
$$


where subscripts $P$ and $t$ refer to $V_{\text {per }}$ and $T_{t}$ parts in Equation (12), respectively, and its components are

$$
\begin{aligned}
\mathcal{W}_{P}= & \sum_{q} \sum_{j \neq 0} k_{q}^{\prime} \frac{1}{2}\left(3 \cos ^{2} \sigma-1\right) \frac{B_{j, q}(I)}{n_{j}} \sin \Theta_{j} \\
& -\frac{1}{2} \sin 2 \sigma \sum_{q} \sum_{j, \rho= \pm 1} k_{p}^{\prime} C_{j, \rho, q}(I) F_{j, \rho}^{1 a} \sin \left(\mu-\rho \Theta_{j}\right) \\
& -\sin \sigma_{c} \frac{M_{c}}{M} \sum_{q} \sum_{j, \rho= \pm 1} k_{p}^{\prime} C_{j, \rho, q}(I) F_{j, \rho}^{2 a} \sin \left(h_{j, \rho}+\nu_{c}\right), \\
\mathcal{W}_{t}= & M \sin \sigma \sum_{q} \sum_{j, \rho= \pm 1} C_{j, \rho, q}(\tilde{I}) D_{j, \rho, q}^{1 a} \sin \left(\tilde{h}_{j, \rho}-\nu\right) \\
& +M_{c} \sin \sigma_{c} \sum_{q} \sum_{j, \rho= \pm 1} C_{j, \rho, q}(\tilde{I}) D_{j, \rho, q}^{2 a} \sin \left(\tilde{h}_{j, \rho}+\nu_{c}\right) .
\end{aligned}
$$

In the former equations $n_{j}=d \Theta_{j} / d t$ are the mean motions of the long-period nutation arguments, and functions $F_{j, \rho}^{k a}, G_{j, \rho}^{k a}$ and $D_{j, \rho, q}^{k a}=k_{q}^{m}\left(F_{j, \rho}^{k a}+G_{j, \rho}^{k a}\right)-k_{q}^{c} G_{j, \rho}^{k a}(k=1,2)$ are the coefficients used in Getino \& Ferrándiz (2001), which are displayed in Appendix A. It can be seen there that coefficients $F$ and $G$ depend on some dimensionless parameters (ellipticities and ratio of inertia moments of Earth and core) and some angular velocities, namely, $\omega_{E}$, the frequencies of the free two-layer Earth wobbles CW and FCN (Chandler Wobble and FCN) and the frequencies of the diurnal band terms in those expansions, $n_{h_{j, \rho}}=d h_{j, \rho} / d t=\omega_{E}-\rho n_{j}$.

The secular second-order term $\mathcal{K}_{2}$ (Equation (16)) can be expressed as

$$
\begin{aligned}
\mathcal{K}_{2}= & \frac{1}{2}\left\{V_{\mathrm{per}}+T_{t}, \mathcal{W}_{P}+\mathcal{W}_{t}\right\}_{\mathrm{sec}} \\
= & \frac{1}{2}\left\{V_{\mathrm{per}}, \mathcal{W}_{P}\right\}_{\mathrm{sec}}+\frac{1}{2}\left\{V_{\mathrm{per}}, \mathcal{W}_{t}\right\}_{\mathrm{sec}} \\
& +\frac{1}{2}\left\{T_{t}, \mathcal{W}_{P}\right\}_{\mathrm{sec}}+\frac{1}{2}\left\{T_{t}, \mathcal{W}_{t}\right\}_{\mathrm{sec}}
\end{aligned}
$$

Therefore, the first step is the calculation of the four Poisson brackets, and then of their secular parts. Notice that in the absence of elasticity, i.e., in Poincaré's Earth model treated in Ferrándiz et al. (2004), only the first bracket was necessary to obtain $\mathcal{K}_{2}$. The main guidelines for computing these Poisson brackets are given in Appendix B. Neglecting the terms of first order in $\sigma$ and $\sigma_{c}$ and higher, we get after some algebra

$$
\begin{aligned}
\left\{V_{\mathrm{per}}, \mathcal{W}_{P}\right\}= & -\frac{1}{M \sin I} \sum_{p, q i, j \neq 0} \frac{k_{p}^{\prime} k_{q}^{\prime}}{n_{j}} B_{i, p} \frac{d B_{j, q}}{d I} m_{5 i} \\
& \times \cos \left(\Theta_{i}-\Theta_{j}\right) \\
& -\frac{1}{M} \sum_{p, q} \sum_{i, j} \sum_{\tau, \rho= \pm 1} k_{p}^{\prime} k_{q}^{\prime} C_{i, \tau, p} C_{j, \rho, q} F_{j, \rho}^{1 a} \\
& \times \cos \left(\tau \Theta_{i}-\rho \Theta_{j}\right) .
\end{aligned}
$$

The first group of terms (coming from zonal-zonal crossing as the presence of the $B_{i}$ coefficients reveals) is analytically the same for rigid one-layer or two-layer Earth models, either rigid (Poincare's) or elastic. The second group, with sectorialsectorial crossing origin shown by coefficients $C_{i}$, is formally kept only inside the class of two-layer models. However, we can recover the rigid Earth expression by replacing $F_{i}^{1 a}$ functions with $1 /\left(n_{\mu}-\tau n_{i}\right)$, where $n_{\mu}=\omega_{E} C / A$.

The remaining Poisson brackets in Equation (21), also truncated at $O\left(\sigma^{0}, \sigma_{c}^{0}\right)$, are

$$
\begin{aligned}
\left\{V_{\mathrm{per}}, \mathcal{W}_{t}\right\}= & \sum_{p, q} \sum_{i, j} \sum_{\tau, \rho= \pm 1} k_{p}^{\prime} D_{j, \rho, q}^{1 a} C_{i, \tau, p} \tilde{C}_{j, \rho, q} \\
& \times \cos \left(\tau \Theta_{i}-\rho \tilde{\Theta}_{j}\right), \\
\left\{T_{t}, \mathcal{W}_{P}\right\}= & \sum_{p, q} \sum_{i, j} \sum_{\tau, \rho= \pm 1} k_{p}^{m} k_{q}^{\prime} F_{j, \rho}^{1 a} \tilde{C}_{i, \tau, p} C_{j, \rho, q} \\
& \times \cos \left(\tau \tilde{\Theta}_{i}-\rho \Theta_{j}\right) \\
& +\frac{M_{c}}{M} \sum_{p, q} \sum_{i, j} \sum_{\tau, \rho= \pm 1} k_{q}^{\prime}\left(k_{p}^{c}-k_{p}^{m}\right) F_{j, \rho}^{2 a} \\
& \times \tilde{C}_{i, \tau, p} C_{j, \rho, q} \cos \left(\tau \tilde{\Theta}_{i}-\rho \Theta_{j}\right), \\
\left\{T_{t}, \mathcal{W}_{t}\right\}= & -M \sum_{p, q} \sum_{i, j} \sum_{\tau, \rho= \pm 1} k_{p}^{m} D_{j, \rho, q}^{1 a} \\
& \times \tilde{C}_{i, \tau, p} \tilde{C}_{j, \rho, q} \cos \left(\tau \tilde{\Theta}_{i}-\rho \tilde{\Theta}_{j}\right) \\
& -M_{c} \sum_{p, q} \sum_{i, j} \sum_{\tau, \rho= \pm 1}\left(k_{p}^{c}-k_{p}^{m}\right) D_{j, \rho, q}^{2 a} \\
& \times \tilde{C}_{i, \tau, p} \tilde{C}_{j, \rho, q} \cos \left(\tau \tilde{\Theta}_{i}-\rho \tilde{\Theta}_{j}\right) .
\end{aligned}
$$

These expressions are exclusively due to the elastic yielding of the two-layer Earth model ( $T_{t}$ term), i.e., they are not present in Poincaré's Earth model (Ferrándiz et al. 2004). They have a double origin. On the one hand, some of them depend indirectly on the core, just through the functions $F_{j, \rho}^{k a}$ and $D_{j, \rho, q}^{k a}$, which are sensitive to the frequency of the NDFW. On the other hand, some terms depend directly on the modulus of the core angular momentum $M_{c}$.

\subsection{Analytical Solution of the Second-order Precession}

The secular part of the Poisson brackets that gives $\mathcal{K}_{2}$ can be obtained straightforwardly, by simply requiring that the angular arguments in Equations (22) and (23) vanish. Omitting the tildes for brevity, in the case of arguments $\Theta_{i}-\Theta_{j}=0$ (zonal-zonal) there is only a possibility, which is satisfied when $i=j$; the contributions to precession of such origin are identified in the following with the superscript 00 . In the most frequent case of having the argument $\tau \Theta_{i}-\rho \Theta_{j}=0$, we have two possibilities: first, $i=j=0$ and unrestricted $\tau, \rho(= \pm 1)$, identified here with the superscript 10; and second, $i=j \neq 0$ and $\tau=\rho$, noted with superscript 11 .

The contributions to the precession rate in longitude derived from the resulting secular part of the first Poisson bracket given in Equation (22) are readily obtained from Equation (15) and 
can be grouped in

$$
\begin{aligned}
\delta p_{P}^{00}= & -\frac{1}{2} \sum_{p, q} \sum_{i \neq 0} \frac{1}{\sin ^{3} I} \frac{k_{p} k_{q}}{n_{i}} m_{5 i} \times\left(\sin I B_{i, p} \frac{d^{2} B_{i, q}}{d I^{2}}\right. \\
& \left.+\sin I \frac{d B_{i, p}}{d I} \frac{d B_{i, q}}{d I}-\cos I B_{i, p} \frac{d B_{i, q}}{d I}\right), \\
\delta p_{P}^{10}= & -\frac{1}{2} \frac{1}{\sin I} \sum_{p, q \tau, \rho= \pm 1} \sum_{p} k_{q} F_{0, \rho}^{1 a} \\
& \times\left(\frac{d C_{0, \tau, p}}{d I} C_{0, \rho, q}+C_{0, \tau, p} \frac{d C_{0, \rho, q}}{d I}\right), \\
\delta p_{P}^{11}= & -\frac{1}{2} \frac{1}{\sin I} \sum_{p, q} \sum_{i \neq 0 \tau= \pm 1} \sum_{p} k_{q} F_{i, \tau}^{1 a} \\
& \times\left(\frac{d C_{i, \tau, p}}{d I} C_{i, \tau, q}+C_{i, \tau, p} \frac{d C_{i, \tau, q}}{d I}\right),
\end{aligned}
$$

$k_{p}=k_{p}^{\prime} / M=k_{p}^{\prime} /\left(C \omega_{E}\right)$ being the usual Kinoshita's (1977) constants of Moon and Sun, proportional to the dynamical ellipticity $H_{d}$.

Let us remark that the $\delta p_{P}^{00}$ term (arising from zonal-zonal crossing) corresponds to the second-order lunisolar effect first computed by Kinoshita \& Souchay (1990), whose value has remained unchanged since then and has been adopted particularly in REN2000 (Souchay et al. 1999), Williams (1994), and Capitaine et al. (2003). Notice that the correspondence is not exact, because the original derivation of it was performed jointly with the $J_{2}$-tilt effect and using a few terms obtained from an elliptical approximation of the Moon and Sun orbits (instead of their own accurate orbital coefficients $A_{i, p}^{(j)}$ ). These three terms were first calculated by Ferrándiz et al. (2004), who used the notation $d p_{0}, d p_{10}, d p_{11}$.

The remaining three Poisson brackets given in Equation (23) provide the contributions steaming from the elasticity of the model and have been derived for the first time in this work, being one of its most important results. They can be arranged as

$$
\begin{aligned}
\delta p_{t, I}^{10}= & \frac{1}{2} \frac{1}{\sin I} \sum_{p, q \tau, \rho= \pm 1} \sum_{p} k_{p}\left[k_{q}^{m}\left(F_{0, \rho}^{1 a}+F_{0, \tau}^{1 a}\right)\right. \\
& \left.+\left(k_{q}^{m}-k_{q}^{c}\right) G_{0, \rho}^{1 a}\right] \frac{d C_{0, \tau, p}}{d I} \tilde{C}_{0, \rho, q}, \\
\delta p_{t, I}^{11}= & \frac{1}{2} \frac{1}{\sin I} \sum_{p, q i \neq 0 \tau \pm 1} \sum_{p} k_{p}\left[k_{q}^{m}\left(F_{i, \tau}^{1 a}+F_{i, \tau}^{1 a}\right)\right. \\
& \left.+\left(k_{q}^{m}-k_{q}^{c}\right) G_{i, \tau}^{1 a}\right] \frac{d C_{i, \tau, p}}{d I} \tilde{C}_{i, \tau, q} . \\
\delta p_{t, D}^{10}= & \frac{1}{2} \frac{1}{\sin I} \frac{M_{c}}{M} \sum_{p, q \tau, \rho= \pm 1} \sum_{q} k_{q}\left(k_{p}^{c}-k_{p}^{m}\right) \\
& \times F_{0, \rho}^{2 a} \frac{d C_{0, \rho, q}}{d I} \tilde{C}_{0, \tau, p}, \\
\delta p_{t, D}^{11}= & \frac{1}{2} \frac{1}{\sin I} \frac{M_{c}}{M} \sum_{p, q i \neq 0 \tau \pm 1} \sum_{i} k_{q}\left(k_{p}^{c}-k_{p}^{m}\right) \\
& \times F_{i, \tau}^{2 a} \frac{d C_{i, \tau, q}}{d I} \tilde{C}_{i, \tau, p} .
\end{aligned}
$$

Superscripts have the same meaning as before and, regarding subscripts, terms $\delta p_{t, I}$ gather the indirect effects of the fluid core, while $\delta p_{t, D}$ contain the direct ones. The latter come from the terms of the generating function that depend on the canonical variables of the core, and are proportional to $M_{c} / M$. Note that the $\left\{T_{t}, \mathcal{W}_{t}\right\}$ bracket does not generate any contribution, since it does not depend on the canonical variable $I$ but on the function of time $\tilde{I}$, so that all the direct effects of the core arise from the second term of $\left\{T_{t}, \mathcal{W}_{P}\right\}$. These effects have not been considered in the literature so far.

The direct and indirect contributions may be added up to a single analytical expression taking into account the following relationship between $F_{i}$ and $G_{i}$ functions:

$$
\frac{M_{c}}{M} F_{i, \tau}^{2 a}=-G_{i, \tau}^{1 a}
$$

which can be obtained from the formulae included in Appendix A. The overall second-order contribution of the elasticity to the precession rate in longitude can thus be written as

$$
\begin{aligned}
\delta p_{t}^{10}= & \frac{1}{2} \frac{1}{\sin I} \sum_{p, q \tau, \rho= \pm 1} \sum_{p} k_{p}\left[k_{q}^{m}\left(F_{0, \rho}^{1 a}+F_{0, \tau}^{1 a}\right)\right. \\
& \left.+2\left(k_{q}^{m}-k_{q}^{c}\right) G_{0, \rho}^{1 a}\right] \frac{d C_{0, \tau, p}}{d I} \tilde{C}_{0, \rho, q} \\
\delta p_{t}^{11}= & \frac{1}{2} \frac{1}{\sin I} \sum_{p, q i \neq 0 \tau= \pm 1} \sum_{p} k_{p}\left[k_{q}^{m}\left(F_{i, \tau}^{1 a}+F_{i, \tau}^{1 a}\right)\right. \\
& \left.+2\left(k_{q}^{m}-k_{q}^{c}\right) G_{i, \tau}^{1 a}\right] \frac{d C_{i, \tau, p}}{d I} \tilde{C}_{i, \tau, q} .
\end{aligned}
$$

These expressions supersede Equation (25). Notice that the indirect effects can be isolated by changing the factor of 2 that multiplies $\left(k_{q}^{m}-k_{q}^{c}\right)$ in Equation (27) to a factor of 1 .

\subsubsection{Final Analytical Expression of the Second-order Contribution}

In conclusion, the total effect $\left.\delta p\right|_{\mathcal{K}_{2}}$ can be represented by means of a sum of three groups of terms in the form

$$
\left.\delta p \equiv \delta p\right|_{\mathcal{K}_{2}}=\delta p_{P}^{00}+\left(\delta p_{P}^{10}+\delta p_{P}^{11}\right)+\left(\delta p_{t}^{10}+\delta p_{t}^{11}\right) .
$$

Each group of terms is associated with certain improvement of the Earth model assumed in the derivation of the second-order effect. A (one-layer) rigid model gives rise to $\delta p_{P}^{00}$ only; a two-layer model with rigid mantle (Poincaré's) adds the terms $\delta p_{P}^{10}+\delta p_{P}^{11}$; finally, $\delta p_{t}^{10}+\delta p_{t}^{11}$ is added in the elastic twolayer Earth case (including both direct and indirect core effects).

\section{NUMERICAL RESULTS AND DISCUSSION}

The analytical expressions that provide the different components of the lunisolar second-order effect have been evaluated numerically. The values assigned to the parameters are displayed in Table 3, together with the sources of each. Parameters specific to the Hamiltonian formulation have been taken from the Getino \& Ferrándiz (2001) two-layer nutation series. Using the values resulting from the fit of a three-layer solution, or a different set of parameters, produces differences below the uncertainty threshold of the determination of precession, given the small magnitude of the effect and the small variations of parameters between both models. At any rate, the analytical nature of our results allows the evaluation of those contributions using different rheological models in a straightforward manner. 
Table 3

Numerical Values of the Parameters Employed in the $\delta p$ Evaluation

\begin{tabular}{llcl}
\hline \hline Parameter & Value & Uncertainty & Source \\
\hline$\omega_{E}$ & $7.292115 \times 10^{-5} \mathrm{rad} \mathrm{s}^{-1}$ & $\ldots$ & Luzum et al. (2011) \\
$I_{0}(\mathrm{~J} 2000)$ & $-84381.406 \mathrm{arcsec}$ & $0.001 \mathrm{arcsec}$ & Luzum et al. (2011) \\
$d \theta / d t$ & $1.00273781191135448 \mathrm{rev}$ UT1-day & \\
$H_{d}$ & $3273795 \times 10^{-9}$ & $\ldots$ & Luzum et al. (2011) \\
$k_{\text {Moon }}$ & $7567.870647 \mathrm{arcsec} \mathrm{cy}^{-1}$ & $1 \times 10^{-9}$ & IERS Conventions Petit \& Luzum (2010) \\
$k_{\text {Sun }}$ & $3474.613747 \operatorname{arcsec~cy}^{-1}$ & $\ldots$ & Getino \& Ferrándiz (2001) \\
$k_{\text {Moon }}^{m}$ & $1906.852345 \operatorname{arcsec~cy}^{-1}$ & $\ldots$ & Getino \& Ferrándiz (2001) \\
$k_{\text {Sun }}^{m}$ & $875.487397 \operatorname{arcsec~cy}^{-1}$ & $\ldots$ & Getino \& Ferrándiz (2001) \\
$k_{\text {Moon }}^{c}$ & $4514.468392 \operatorname{arcsec~cy}^{-1}$ & $\ldots$ & Getino \& Ferrándiz (2001) \\
$k_{\text {Sun }}^{c}$ & $2072.714329 \operatorname{arcsec~cy}^{-1}$ & $\ldots$ & Getino \& Ferrándiz (2001) \\
$P_{\mathrm{CW}}$ & $430.9 \mathrm{msd}$ & $0.7 \mathrm{msd}$ & Getino \& Ferrándiz (2001) \\
$P_{\mathrm{FCN}}$ & $430.00 \mathrm{msd}$ & $0.01 \mathrm{msd}$ & Nastula \& Gross (2015) \\
$A_{c} / A_{m}$ & 0.1284 & $\ldots$ & Krásná et al. (2013) \\
Orbital coefficients & Getino et al. (2010), Table 8 & $\ldots$ & Dziewonski \& Anderson (1981) \\
\hline
\end{tabular}

Note. The ellipsis points signify that the uncertainty is not provided in the source along with the value. The $H_{d}$ value and uncertainty in IERS Conventions (Petit \& Luzum 2010) are consistent with the IAU2006/2000 precession-nutation model (Capitaine et al. 2003).

Table 4 contains the numerical values of the different individual components of the precession rate contribution derived in Section 2.4 for the considered elastic two-layer model. The three central rows correspond to the different kinds of groups identified with superscripts according to their origin, 00 (zonal) and 10, 11 (sectorial), whose constituents were distinguished with subscripts $p, t$. The last two groups arise from the components of the Hamiltonian and the generating function, which give rise to the Oppolzer nutation terms at first order. These kinds of terms were introduced in the precession theory by Ferrándiz et al. (2004). Their magnitude is strongly dependent on the Earth model features, since they are amplified by the resonance induced by the fluid core as happens with Oppolzer terms in nutations.

The first column of figures (column (2)) provides the first three terms, $\delta p_{P}^{00}, \delta p_{P}^{10}$, and $\delta p_{P}^{11}$, of the total contribution shown in Equation (28). Let us recall that their sum would contain the total second-order effect if we removed elasticity from the hypothesis; the column is labeled as Poincaré-rigid mantle + fluid core for that reason. Besides, the first term of column (2), $\delta p_{P}^{00}$, is common to rigid and non-rigid Earth solutions; it is obtained from the terms of the potential and of the first-order generating function that produce the Poisson nutation terms at first order (Kinoshita 1977). The next two columns display the additional contributions stemming from elasticity, named $\delta p_{t}^{10}$ and $\delta p_{t}^{11}$ in Equation (28), separating in each the indirect (column (3)) and direct (column (4)) effects of the core, defined in Equation (25)- $\delta p_{t, I}^{1 x}$ and $\delta p_{t, D}^{1 x}$, respectively. The last column sums the contributions of each kind, and the last row is the total by columns.

Let us remark that all the constituents of the total precession rate (except one, the direct core effect $\delta p_{t, D}^{11}$ ) exceed 0.3 mas $/ \mathrm{cy}$. We have taken here that value as proxy of the current accuracy needs because it is the GGOS stability goal and also the estimation of the speed of the general precession in longitude made by Fukushima (2003). It can also be seen that the direct effects of the core are smaller than but reach the same order of magnitude as the indirect ones. Looking at the last column, which gathers the total contribution of each group, we can consider that its first cell, $-45.51 \mathrm{mas} / \mathrm{cy}$, has been somehow accounted for in precession theory, since it corresponds to an
Table 4

Second-order Lunisolar Contributions to Precession Rate in Longitude (mas/ cy) for the Elastic Two-layer Earth Model Considered in This Work

\begin{tabular}{lcccc}
\hline \hline & Poincaré's Model & \multicolumn{2}{c}{ Elasticity } & \\
\hline Additive & Rigid Mantle & \multicolumn{2}{c}{ Core Effects } & TOTAL \\
\cline { 3 - 4 } contributions & + Fluid Core & Indirect & Direct & \\
\hline$\delta p^{00}$ & -45.51 & & & -45.51 \\
$\delta p^{10}$ & -22.43 & +9.45 & +3.80 & -9.17 \\
$\delta p^{11}$ & -1.26 & +0.48 & +0.17 & -0.61 \\
\hline$\delta p$ & -69.20 & +9.94 & +3.97 & -55.29 \\
& & & Uncertainty ${ }^{\mathrm{a}}:$ & \pm 0.01 \\
\hline
\end{tabular}

Note.

${ }^{\mathrm{a}}$ See the text for discussion.

effect ( $\delta p^{00}$ term) common to rigid and non-rigid Earth models, as noted in the previous paragraph. However, the contributions in the other two cells (which gather the total $\delta p^{10}$ and $\delta p^{11}$ effects of sectorial origin) have never been considered in IAU precession theories so far. These last effects amount to -9.78 mas/cy, about $20 \%$ of the zonal, rigid effect accounted for so far. Let us notice too that in the absence of elasticity the sectorial terms would produce a much larger effect that reaches $-23.67 \mathrm{mas} / \mathrm{cy}$; therefore, elasticity reduces the impact of the NDFW resonance in the precession rate in the same way that it happens in nutation theory.

To ascertain the uncertainty of our evaluation to -55.29 mas/cy of the second-order effect is not simple, and any estimation of it has its own drawbacks. Anyway, as it follows from an approximate analytical solution of a given model, the discussion can be started by grouping the sources of inaccuracy into two classes: one relative to the goodness of the approximation provided by that mathematical solution, and the other associated with the inaccuracies of the values of the astronomical and geophysical parameters used in the numerical evaluation of the analytical solution. The first can be ignored since the error of our solution is of the third order 
Table 5

Comparison of Second-Order Corrections ( $\delta p$ in mas/cy)

\begin{tabular}{|c|c|c|c|c|}
\hline Solution & IAU2006 & RDAN97 & FNEG04 & This Work \\
\hline$\delta p^{00}$ & -46.78 & -45.40 & -45.36 & -45.51 \\
\hline$\delta p^{11}$ & & & -1.30 & -0.61 \\
\hline Total $\delta p$ & -46.78 & -45.40 & -69.25 & -55.29 \\
\hline IAU2006 value & 0 & +1.38 & -22.47 & -8.51 \\
\hline Associated $\delta H_{d}$ & 0 & $-90 \times 10^{-11}$ & $1459 \times 10^{-11}$ & $553 \times 10^{-11}$ \\
\hline$H_{d, \mathrm{IAU} 2006}+\delta H_{d}$ & 0.00327379448 & 0.00327379358 & 0.00327380907 & 0.00327380001 \\
\hline Relative $H_{d}$ change (ppm) & 0 & -0.3 & +4.5 & +1.7 \\
\hline
\end{tabular}

of perturbation, and the dimensionless perturbation order is $O\left(\left(V_{\text {sec }}+V_{\text {per }}\right) / T_{0}\right) \approx 10^{-7}$ (see, e.g., Getino \& Ferrándiz 1995).

The second kind of error is more intricate, since the uncertainties of many parameters are not provided in the original references and seem to be unavailable in the literature. It forces us to make complementary assumptions.

For instance, the first component $\delta p^{00}$ is independent of the Earth model as noticed before; although the notation may not be revealing enough, it only depends on a few astronomical parameters, basically those present in Equation (2), all of which are known with a relative uncertainty better than $10^{-6}$. Therefore, all four significant figures of its value displayed in Table 4 can be taken as exact if this reasoning is considered plausible.

The two remaining components, $\delta p^{10}$ and $\delta p^{11}$, depend also on additional geophysical parameters, mainly the periods of the Chandler wobble and the retrograde $\mathrm{FCN}, P_{\mathrm{CW}}$ and $P_{\mathrm{FCN}}-$ which have been widely studied-and the ratio $r_{c m}=A_{c} / A_{m}$. If we adopt for $P_{\mathrm{CW}}$ and $P_{\mathrm{FCN}}$ the values and uncertainties provided in Table 3 , the resulting $\delta p$ uncertainty evaluates to $\pm 0.002 \mathrm{mas} / \mathrm{cy}$, and those parameters would have no effect on the displayed figures.

The $r_{\mathrm{cm}}$ ratio is the most critical parameter. Although there is a high degree of agreement among different determinations of it, we found no recent, reliable, explicit evaluation of its uncertainty and had to resort to indirect estimations. For instance, we could consider the recent determinations of the moments of inertia of Earth's layers appearing in Table 9 of Chen et al. (2015), which assimilate information from the most accurate geopotential models. The $r_{\mathrm{cm}}$ uncertainty estimated from those data is \pm 0.00005 and contributes with \pm 0.006 mas / cy to the $\delta p$ uncertainty, whose final value has been set to $\pm 0.01 \mathrm{mas} / \mathrm{cy}$ in Table 4 . A more conservative estimation of the $r_{\mathrm{cm}}$ influence can be derived from the difference between their values derived from PREM and 1066A models (see, e.g., Table 1 of Mathews et al. 1991). The uncertainties of $r_{\mathrm{cm}}$ and $\delta p$ are then estimated to be \pm 0.0008 and $\pm 0.06 \mathrm{mas} / \mathrm{cy}$, respectively.

Therefore, the $r_{\mathrm{cm}}$ ratio is the dominant source of uncertainty under both assumptions, and the respective values, 0.01 and $0.06 \mathrm{mas} / \mathrm{cy}$, have the same order of magnitude. In conclusion, our correction can be considered more accurate than the determination of the precession rate cited in the introduction even under the less favorable assumption.
In Table 5 our solution is compared to previous determinations of this second-order effect made for simpler Earth models. Column (2), with the IAU2006 heading, shows the value $-46.78 \mathrm{mas} / \mathrm{cy}$ given to this correction in the P03 theory, the sum of the second-order lunisolar effects (a) and (b) in Capitaine et al. (2003) (Table 3). That value was first derived by Kinoshita \& Souchay (1990) and kept unchanged by Williams (1994), Souchay \& Kinoshita (1996), and in the final release of REN2000 (Souchay et al. 1999) as well. The original derivation only took into account four zonal terms and used an elliptical approximation for the orbits of the Moon and Sun. It is remarkable that their value only deviates $1 \%$ from our complete computation in the rigid Earth case, which includes 198 terms with the same orbits used for the first-order nutations and arrives at $-45.51 \mathrm{mas} / \mathrm{cy}$. In fact, an evaluation with a reduced set of the largest 11 terms (Getino et al. 2010) produces no significant variations. The value obtained by Roosbeek \& Dehant (1998) in their rigid Earth RDAN97 theory appears in column (3) and is very close to ours.

Column (4) displays the results published for a two-layer Poincaré Earth model FNEG04 (Ferrándiz et al. 2004) with a total amount of $-69.25 \mathrm{mas} / \mathrm{cy}$, close to the recomputation of this work (see Table 4, column (2)). The small difference is due to the different set of parameters used in both investigations, $P_{\mathrm{CW}}$ and $P_{\mathrm{FCN}}$ in particular. Although for the sake of simplicity they are not displayed in Table 5, the results derived in this work are also consistent with the preliminary computations of the indirect terms for a similar two-layer Earth model (Ferrándiz et al. 2007), also shown in Table 4. Regarding the rigid case, the first detailed derivation of the sectorial contribution to the second-order effect on the longitude rate appeared jointly with the second-order nutation solution by Getino et al. (2010), who obtained -0.69 mas/cy, very close to the $-0.70 \mathrm{mas} / \mathrm{cy}$ here. The row below the total value of each solution provides the respective deviations with respect to the IAU2006 reference correction (i.e., the REN2000 value). It is readily seen that all the deviations exceed $0.3 \mathrm{mas} / \mathrm{cy}$ and are thus above the current goals of accuracy of Earth rotation theories cited in Section 1.

The first-order precession rate $p_{A}^{\prime}$ is estimated by subtracting the corrections recognized in the relevant theory from the observed rate, and then the dynamical ellipticity is determined from $p_{A}^{\prime}$ taking into account Equation (2) (maybe in the context of a wider parameter fitting). Therefore, any change $\delta p$ of the second-order correction with respect to its reference value in 
IAU2006 must be compensated by an equal and opposite change $\delta p_{A}^{\prime}=-\delta p$ of the first-order rate $p_{A}^{\prime}$ to keep the total rate unchanged. In turn, that variation induces an associated variation $\delta H_{d}$ of the dynamical ellipticity such that $\delta H_{d}=$ $\left(H_{d} / p_{A}^{\prime}\right) \delta p$ and requires a rescaling of the nutation series (Escapa et al. 2016).

The next row of Table 5 displays the variations in each case. The uncertainties of the implied $H_{d}$ variations can be estimated similarly. Taking into account the pair of former possible values of the $\delta p$ uncertainty, the $\delta H_{d}=553 \times 10^{-11}$ induced by the $\delta p$ deviation $-8.51 \mathrm{mas} / \mathrm{cy}$ can be attributed to an uncertainty of $\pm 0.4 \times 10^{-11}$ (and below $\pm 3.9 \times 10^{-11}$ in any case). It is worth underlining that this value is more than four times larger than the uncertainty in the estimation of $H_{d}$ made by MHB2000, which was $1.2 \times 10^{-9}$, equivalent to $0.4 \mathrm{ppm}$.

The results of applying the former corrections to the reference value $H_{d}=0.00327379448$ adopted in IAU2006 (Capitaine et al. 2003, 2009) are shown in the next row. Note that the uncertainty of the $\delta H_{d}$ corrections should not be extrapolated to the modified values of $H_{d}$ since the main source of error of $p_{A}^{\prime}$ still comes from the determination of the observed rate. Finally, the relative variations of the modified $H_{d}$ values with respect to the reference one are provided in ppm in the last row. This makes the estimation of the magnitude of the corrections to the main nutation terms easy, taking $17^{\prime \prime}$ and $9^{\prime \prime}$ as proxies of maximum longitude and obliquity amplitudes.

\section{CONCLUSIONS}

The contribution to the precession rate due to the secondorder solution associated with the main lunisolar potential has been considered in precession theory for decades; it is the third in magnitude, after the geodesic precession and the $J_{2}-$ tilt rates. The IAU2006 precession theory adopted for it the value -46.78 mas/cy, corresponding to a simplified rigid Earth model and derived by means of Hamiltonian perturbation methods. In this paper we have carried out the computation of that effect in the Hamiltonian framework, assuming a two-layer Earth model and linear elasticity, and have arrived at an analytical expression to evaluate those contributions. They provide a final value of $-55.29 \mathrm{mas} / \mathrm{cy}$. The difference between both values amounts to $-8.51 \mathrm{mas} / \mathrm{cy}$ and is noticeably larger than the $-0.3 \mathrm{mas} / \mathrm{cy}$ that can be taken as a target of precession theory considering the observational accuracy and the GGOS goals for reference frames. Therefore, the value of this correction must be updated and the dynamical ellipticity of Earth revised accordingly.

This research has been partially supported by the Spanish government MINECO projects AYA2010-22039-C02-02 and AYA2016-79775-P (AEI/FEDER, UE). The authors acknowledge the valuable advice of an anonymous referee that helped to improve the manuscript.

\section{APPENDIX A \\ FUNCTIONS APPEARING IN THE PRECESSION FORMULAE}

The functions $B_{i, p}, C_{i, \tau, p}$, and $D_{i, \tau, p}$ are those defined in Kinoshita (1977) and depend on the orbital coefficients $A_{i, p}^{(j)}$ provided in Kinoshita \& Souchay (1990) or Navarro (2001).
Their expressions are

$$
\begin{aligned}
B_{i, p}(I)= & -\frac{1}{6}\left(3 \cos ^{2} I-1\right) A_{i, p}^{(0)}-\frac{1}{2} \sin 2 I A_{i, p}^{(1)} \\
& -\frac{1}{4} \sin ^{2} I A_{i, p}^{(2)}, \\
C_{i, \tau, p}(I)= & -\frac{1}{4} \sin 2 I A_{i, p}^{(0)}+\frac{1}{2}(1+\tau \cos I) \\
& \times(-1+2 \tau \cos I) A_{i, p}^{(1)} \\
& +\frac{1}{4} \tau \sin I(1+\tau \cos I) A_{i, p}^{(2)}, \\
D_{i, \tau, p}(I)= & -\frac{1}{2} \sin ^{2} I A_{i, p}^{(0)}+\tau \sin I(1+\tau \cos I) A_{i, p}^{(1)} \\
& -\frac{1}{4}(1+\tau \cos I)^{2} A_{i, p}^{(2)} .
\end{aligned}
$$

The two-layer Earth nutation solution by Getino \& Ferrándiz (2001) is given in terms of a set of functions that allows determining the amplitudes of the nutation series. When particularizing them to the Earth model considered in this work $(\Gamma=0)$, we have

$$
F_{i, \tau}^{1 a}=\frac{r_{4}+n_{h_{i, \tau}}}{f_{1 ; i, \tau} f_{2 ; i, \tau}}, F_{i, \tau}^{2 a}=\frac{r_{2}}{f_{1 ; i, \tau} f_{2 ; i, \tau}}, \quad G_{i, \tau}^{1 a}=\frac{r_{3}}{f_{1 ; i, \tau} f_{2 ; i, \tau}},
$$

with

$$
f_{1 ; i, \tau}=m_{1}+\omega_{E}-\tau n_{i}, f_{2 ; i, \tau}=m_{2}+\omega_{E}-\tau n_{i}
$$

These expressions can be written (Getino \& Ferrándiz 2001) in terms of the BEP of the model $\left\{P_{\mathrm{CW}}, P_{\mathrm{FCN}}, A_{c} / A_{m}\right\}$ as

$$
\begin{aligned}
r_{2} & =-\omega_{E}\left(1+\frac{A_{c}}{A_{m}}+\frac{1}{P_{\mathrm{CW}}}\right), \\
r_{3} & =\omega_{E} \frac{\left(A_{c} / A_{m}\right)}{1+\left(A_{c} / A_{m}\right)}\left(1+\frac{A_{c}}{A_{m}}+\frac{1}{P_{\mathrm{FCN}}}\right), \\
r_{4} & =-\omega_{E}\left(1+\frac{A_{c}}{A_{m}}+\frac{1}{P_{\mathrm{FCN}}}\right), \\
m_{1} & =\frac{\omega_{E}}{P_{\mathrm{CW}}} \\
m_{2} & =-\omega_{E}\left(1+\frac{1}{P_{\mathrm{FCN}}}\right)
\end{aligned}
$$

where $P_{\mathrm{CW}}$ is associated with the Chandler wobble, $P_{\mathrm{FCN}}$ is associated with the FCN, and $A_{c} / A_{m}$ is the ratio between the equatorial moment of inertia of the core and the mantle.

\section{APPENDIX B \\ COMPUTATION OF POISSON BRACKETS}

In the notation we follow, the Poisson bracket of two smooth functions $f$ and $g$ of the canonical set $(q, p)$, is defined by the bilinear operation

$$
\{f(q, p), g(q, p)\}=\sum_{i=1}^{n}\left(\frac{\partial f}{\partial q_{i}} \frac{\partial g}{\partial p_{i}}-\frac{\partial f}{\partial p_{i}} \frac{\partial g}{\partial q_{i}}\right) .
$$


In terms of the Andoyer-like canonical set of a two-layer Earth, the Poisson bracket is explicitly written as

$$
\begin{aligned}
\{f, g\}= & \frac{\partial f}{\partial \mu} \frac{\partial g}{\partial M}-\frac{\partial f}{\partial M} \frac{\partial g}{\partial \mu}+\frac{\partial f}{\partial \nu} \frac{\partial g}{\partial N}-\frac{\partial f}{\partial N} \frac{\partial g}{\partial \nu} \\
& +\frac{\partial f}{\partial \lambda} \frac{\partial g}{\partial \Lambda}-\frac{\partial f}{\partial \Lambda} \frac{\partial g}{\partial \lambda}+\frac{\partial f}{\partial \mu_{c}} \frac{\partial g}{\partial M_{c}}-\frac{\partial f}{\partial M_{c}} \frac{\partial g}{\partial \mu_{c}} \\
& +\frac{\partial f}{\partial \nu_{c}} \frac{\partial g}{\partial N_{c}}-\frac{\partial f}{\partial N_{c}} \frac{\partial g}{\partial \nu_{c}}+\frac{\partial f}{\partial \lambda_{c}} \frac{\partial g}{\partial \Lambda_{c}}-\frac{\partial f}{\partial \Lambda_{c}} \frac{\partial g}{\partial \lambda_{c}} .
\end{aligned}
$$

The former expression can be split into two parts formally identical to two Poisson brackets that would depend only on the subset of variables corresponding to the whole Earth, $\mathcal{S}_{E}=(\lambda, \mu, \nu, \Lambda, M, N)$, and that of the core, $\mathcal{S}_{c}=$ $\left(\lambda_{c}, \mu_{c}, \nu_{c}, \Lambda_{c}, M_{c}, N_{c}\right)$,

$$
\{f, g\}=\{f, g\}_{E}+\{f, g\}_{c},
$$

denoted with $E$ and $c$ subscripts.

In our developments the functions $f$ and $g$ may depend on the auxiliary angles $\sigma, \sigma_{c}, I$, and $I_{c}$, defined through

$$
\cos \sigma=\frac{N}{M}, \cos I=\frac{\Lambda}{M}, \cos \sigma_{c}=\frac{N_{c}}{M_{c}}, \cos I_{c}=\frac{\Lambda_{c}}{M_{c}} .
$$

Hence, the derivatives with respect to canonical momenta must be performed by means of the following expressions (chain rule):

$$
\begin{aligned}
\frac{\partial}{\partial M} & =\left(\frac{\partial}{\partial M}\right)+\frac{\cot \sigma}{M} \frac{\partial}{\partial \sigma}+\frac{\cot I}{M} \frac{\partial}{\partial I}, \\
\frac{\partial}{\partial N} & =\left(\frac{\partial}{\partial N}\right)-\frac{1}{M \sin \sigma} \frac{\partial}{\partial \sigma}, \\
\frac{\partial}{\partial \Lambda} & =\left(\frac{\partial}{\partial \Lambda}\right)-\frac{1}{M \sin I} \frac{\partial}{\partial I},
\end{aligned}
$$

and the analogous ones for the core variables ( $c$ subscript). The derivatives in parentheses refer to partial derivatives in the case of explicit dependence of the related variables.

Computing the Poisson brackets with the assistance of a symbolic processor is a good option to avoid annoying calculations and decrease the risk of flaws. The brackets in Section 2.3 were derived in this way, with the aid of Maple software. If hand calculation is preferred or used for checking purposes, we can tackle the advantage of the particular functional dependence of $V_{\mathrm{per}}, T_{t}, \mathcal{W}_{P}$, and $\mathcal{W}_{t}$-Equations (17) and (20)—functions to simplify the process.

First, $\sigma$ and $\sigma_{c}$ angles are of small magnitude, about $10^{-6} \mathrm{rad}$ (see, e.g., Getino 1995). Therefore, once the Poisson brackets have been computed, we can ignore their terms beyond the zeroth order in $\sigma$ and $\sigma_{c}$, since we only need to compute the partial derivative with respect to $I$ according to Equation (15). Furthermore, most of the brackets in Equation (21) can be computed simply as reduced $\{-,-\}_{E}$ or $\{-,-\}_{c}$ brackets, since only the third term in $\mathcal{W}_{P}$ given in Equation (20) depends on $\mathcal{S}_{c}$ variables and also on a sole $\mathcal{S}_{E}$ variable, $I$.
Finally, the functional dependence of all the terms involved in the computation (except the one just mentioned) allows them to be arranged in one of the following forms:

$$
\begin{aligned}
& f_{0}=S_{0} V_{0}(I, \lambda), g_{0}=S_{0} \mathcal{W}_{0}(I, \lambda), \\
& f_{1}=S_{1} V_{1}(I, \lambda, \mu, \nu), g_{1}=S_{1} \mathcal{W}_{1}(I, \lambda, \mu, \nu),
\end{aligned}
$$

with $S_{0}=3 \cos ^{2} \sigma-1, S_{1}=\sin \sigma \cos \sigma$, and similarly for the core ( $c$ subscript).

For instance, the computation of the bracket $\left\{V_{\mathrm{per}}, \mathcal{W}_{P}\right\}$ of Equation (22) reduces to the form $\left\{f_{0}+f_{1}, g_{0}+g_{1}\right\}_{E}$, which produces few nonvanishing terms. Specifically, we have

$$
\begin{aligned}
\left\{f_{0}, g_{0}\right\}_{E}= & \frac{4}{M \sin I}\left[-\frac{\partial V_{0}}{\partial \lambda} \frac{\partial \mathcal{W}_{0}}{\partial I}+\frac{\partial V_{0}}{\partial I} \frac{\partial \mathcal{W}_{0}}{\partial \lambda}\right]+O(\sigma), \\
\left\{f_{0}, g_{1}\right\}_{E}= & \left\{f_{1}, g_{0}\right\}_{E}=0+O(\sigma), \\
\left\{f_{1}, g_{1}\right\}_{E}= & \frac{1}{M}\left[\left(\frac{\partial V_{1}}{\partial \mu}-\frac{\partial V_{1}}{\partial \nu}\right) \mathcal{W}_{1}-\left(\frac{\partial \mathcal{W}_{1}}{\partial \mu}-\frac{\partial \mathcal{W}_{1}}{\partial \nu}\right) V_{1}\right] \\
& +O(\sigma) .
\end{aligned}
$$

The remaining Poisson brackets of Section 2.3 can be derived in a similar way.

\section{REFERENCES}

Capitaine, N., Mathews, P. M., Dehant, V., Wallace, P. T., \& Lambert, S. B. 2009, CeMDA, 103, 179

Capitaine, N., Wallace, P. T., \& Chapront, J. 2003, A\&A, 412, 567

Capitaine, N., Wallace, P. T., \& Chapront, J. 2005, A\&A, 432, 355

Chen, W., Li, J. C., Ray, J., Shen, W. B., \& Huang, C. L. 2015, JGeod, 89, 179

Dziewonski, A. M., \& Anderson, D. L. 1981, PEPI, 25, 297

Efroimsky, M., \& Williams, J. G. 2009, CeMDA, 104, 257

Escapa, A. 2011, CeMDA, 110, 99

Escapa, A., Ferrándiz, J. M., Baenas, T., et al. 2016, PApGe, 173, 861

Ferrándiz, J. M., \& Gross, R. S. 2015, in The New IAU/IAG Joint Working Group on Theory of Earth Rotation, International Association of Geodesy Symposia Ser. Vol. 143 (New York: Springer), 543

Ferrándiz, J. M., Navarro, J. F., Escapa, A., \& Getino, J. 2004, AJ, 128, 1407

Ferrándiz, J. M., Navarro, J. F., Escapa, A., Getino, J., \& Baenas, T. 2007, Influence of the Core on the Earth Precession, Proc. Workshop Organized in Honour of Professor Jacques Henrard at the Occasion of His Retirement (Presses Universitaires of Namur), 9

Ferraz-Mello, S. 2007, Canonical Perturbation Theories. Degenerate Systems and Resonance (Berlin: Springer)

Folgueira, M., Bizouard, C. H., \& Souchay, J. 2001, CeMDA, 81, 191

Folgueira, M., Souchay, J., \& Kinoshita, H. 1998, CeMDA, 69, 373

Folgueira, M., Souchay, J., \& Kinoshita, H. 1999, CeMDA, 70, 147

Fukushima, T. A. 2003, AJ, 126, 494

Getino, J. 1995, GeoJI, 122, 803

Getino, J., Escapa, A., \& Miguel, D. 2010, AJ, 139, 1916

Getino, J., \& Ferrándiz, J. M. 1991, CeMDA, 51, 35

Getino, J., \& Ferrándiz, J. M. 1995, CeMDA, 61, 117

Getino, J., \& Ferrándiz, J. M. 1997, GeoJI, 130, 326

Getino, J., \& Ferrándiz, J. M. 2001, MNRAS, 322, 785

Hilton, J. L., Capitaine, N., Chapront, J., et al. 2006, CeMDA, 94, 351

Hori, G. I. 1966, PASJ, 18, 287

Kaula, W. M. 1964, RvGSP, 2, 661

Kinoshita, H. 1977, CeMDA, 15, 277

Kinoshita, H., \& Souchay, J. 1990, CeMDA, 48, 187

Krásná, H., Böhm1, J., \& Schuh, H. 2013, A\&A, 555, A29

Kubo, Y. 1991, CeMDA, 50, 165

Lambert, S. B., \& Mathews, P. M. 2006, A\&A, 453, 363

Lieske, J. H., Lederle, T., Fricke, W., \& Morando, B. 1977, A\&A, 58, 1

Liu, J.-C., \& Capitaine, N. 2015, in Proc. Journeés 2014 Syst. Ref. Possible Improvements of the IAU 2006 Precession Based on Recent Progress, ed. Z. Malkin \& N. Capitaine (St. Petersburg: Pulkovo Observatory), 155 
Luzum, B., Capitaine, N., Fienga, A., et al. 2011, CeMDA, 110, 293

Mathews, P. M., Buffet, B. A., Herring, T. A., \& Shapiro, I. 1991, JGR, 96, 8243

Mathews, P. M., Herring, T. A., \& Buffet, B. A. 2002, JGR, 107, 2068

Nastula, J., \& Gross, R. 2015, JGRB, 120, 4474

Navarro, J. F. 2001, PhD thesis, Univ. Alicante

Petit, G., \& Luzum, B. (ed.) 2010, IERS Conventions (2010), IERS Technical Note 36 (Verlag des Bundesamtes für Kartographie und Geodäsie, Frankfurt am Main)
Plag, H., \& Pearlman, M. 2009, Global Geodetic Observing System (Berlin: Springer)

Roosbeek, F., \& Dehant, V. 1998, CeMDA, 70, 215

Souchay, J., \& Kinoshita, H. 1996, A\&A, 312, 1017

Souchay, J., Losley, B., Kinoshita, H., \& Folgueira, M. 1999, A\&AS, 135,111

Vondrák, J., Capitaine, N., \& Wallace, P. 2011, A\&A, 534, 1

Williams, J. G. 1994, AJ, 108, 711

Williams, J. G. 1995, AJ, 110, 1420 